Research Article

\title{
Threshold Estimation for a Spectrally Negative Lévy Process
}

\author{
Honglong You $(\mathbb{D})$ and Chuncun Yin (1D) \\ School of Statistics, Qufu Normal University, Jining, Shandong 273165, China \\ Correspondence should be addressed to Honglong You; youhonglong815@163.com
}

Received 11 June 2020; Accepted 7 July 2020; Published 30 July 2020

Guest Editor: Wenguang Yu

Copyright () 2020 Honglong You and Chuncun Yin. This is an open access article distributed under the Creative Commons Attribution License, which permits unrestricted use, distribution, and reproduction in any medium, provided the original work is properly cited.

\begin{abstract}
Consider a spectrally negative Lévy process with unknown diffusion coefficient and Lévy measure and suppose that the high frequency trading data is given. We use the techniques of threshold estimation and regularized Laplace inversion to obtain the estimator of survival probability for a spectrally negative Lévy process. The asymptotic properties are given for the proposed estimator. Simulation studies are also given to show the finite sample performance of our estimator.
\end{abstract}

\section{Introduction}

In actuarial science, it is an important topic to consider the ruin probability for some risk models. There are some methods for this topic, for example, the integro-differential equation technique, renewal theory, Laplace transform, martingale theory, and so on. For details, see the monograph of Asmussen and Albrecher [1]. These methods heavily depend on the knowledge of the risk model, which are usually unknown in practice. It is also known that explicit formula for the ruin probability is usually not available when we have no precise information on the risk model. In order to overcome this difficulty, many researchers have done a large amount of work and obtained lots of nice results. Some authors have considered the approximations, upper and lower bounds of the ruin probability. See, for example, Dufresne and Gerber [2], Veraverbeke [3], Dermitzakis and Politis [4], and Li et al. [5]. Others have been contributed to semiparametric and nonparametric estimation of the ruin probability. See, for example, Croux and Veraverbeke [6], Frees [7], Mnatsakanov et al. [8], Pitts [9], Politis [10], You et al. [11], and Zhang et al. [12].

In practical situations, to get the data is much easier than to obtain the precise information on the risk model. In financial market, high frequency trading exists and a lot of high frequency trading data can be used to make statistical inference of the law of financial market. Using the data, we can estimate the survival probability by some statistical methods. In our work, we assume that the high frequency data is from $n$ discrete time observations with step $h_{n}$. The asymptotic framework is that $n$ tends to infinity and $h_{n}$ tends to zero while $n h_{n}$ tends to infinity. See, Comte and GenonCatalot $[13,14]$. For an insurance company, if the surplus has lots of small fluctuations, we assume that the surplus may be described by Lévy process. Our work will consider the survival probability for Lévy process. There are also some nice results for the ruin probability in Lévy process. For example, Zhang and Yang [15] have proposed a nonparametric estimator of ruin probability for pure jump Lévy process by Fourier (inversion) transform. The method of Zhang and Yang [15] has also been used by Shimizu and Zhang [16] to study the Gerber-Shiu function for Lévy subordinator. You and Cai [17] and Cai et al. [18] have constructed an estimator for the survival probability in a spectrally negative Lévy risk model by the regularized Laplace inversion technique. In the paper, we will use a threshold technique to study the survival probability. Mancini [19], Mancini [20], Shimizu [21], and Shimizu [22] have proposed the threshold technique for identifying the times when jumps larger than a suitably defined threshold occurred. Given a discrete record of observations, the technique may separate the contributions of the diffusion part and jump part of the risk model. Therefore, we can obtain more accurate data for the jump part of the risk 
model by the threshold technique. In this paper, we will give an estimator of the survival probability by the threshold technique and the regularized Laplace inversion technique. Our method will calculate the survival probability more accurately for a spectrally negative Lévy risk model.

Here is a brief outline of this paper. We will introduce our risk model and define the survival probability in Section 2. In Section 3, we will construct an estimator of survival probability for our risk model. Section 4 gives the asymptotic properties of the estimators. In Section 5, we will do some simulations to show the finite sample size performance of the estimators. Finally, some conclusions are given in Section 6. All the technical proofs are presented in Appendix.

\section{Preliminaries}

2.1. Risk Model. Let

$$
Y_{t}=c t+\sigma W_{t}-J_{t}, \quad t \geq 0
$$

be a spectrally negative Lévy process, where $c>0, \sigma>0$, and $W=\left\{W_{t}, t \geq 0\right\} \quad$ is a standard Brownian motion; $J=\left\{J_{t}, t \geq 0\right\}$ is a subordinator; Suppose that $W$ and $J$ are independent of each other.

The Laplace exponent of $Y=\left\{Y_{t}, t \geq 0\right\}$ is denoted by

$$
\begin{aligned}
\psi_{Y}(s) & =\frac{1}{t} \ln \left(\mathbf{E}\left[e^{s Y_{t}}\right]\right) \\
& =c s+\frac{1}{2} \sigma^{2} s^{2}-\int_{0}^{\infty}\left(1-e^{-s x}\right) \nu(\mathrm{d} x), \quad s>0,
\end{aligned}
$$

where $v$ is a Lévy measure and satisfies $\int_{0}^{\infty}(1 \wedge x)(\mathrm{d} x)<\infty$.

Let $u>0$ be the initial surplus of an insurance company. The surplus at time $t$ is given by

$$
U_{t}=u+Y_{t}=u+c t+\sigma W_{t}-J_{t}, \quad t \geq 0,
$$

where $c$ is the rate of premium; $\sigma$ represents the diffusion coefficient; and $J$ and $W$ denote the cumulative claims amount and a diffusion process.

2.2. Survival Probability. The infinite-time horizon survival probability $\Phi(u)$ is defined as follows:

$$
\Phi(u)=1-\mathbf{P}\left(\inf _{0 \leq t<\infty} U_{t} \leq 0 \mid U_{0}=u\right) .
$$

In [23], Huzak et al. have given the following Pollaczek-Khinchin type formula for the survival probability,

$$
\Phi(u)=\left(1-\frac{\mu_{1}}{c}\right) \sum_{i=0}^{\infty} \rho^{i}\left(G^{(i+1) *} * H^{(i) *}\right)(u), \quad u>0
$$

where $\mu_{1}=\int_{0}^{\infty} x v(\mathrm{~d} x), H(x)=\left(1 / \mu_{1}\right) \int_{0}^{x} \nu(y, \infty) \mathrm{d} y$ and $G$ is determined by the Laplace transform

$$
\int_{0}^{\infty} e^{-s x} \mathrm{~d} G(x)=\frac{c}{c+\left(\sigma^{2} / 2\right) s} .
$$

By (5), the Laplace transform of $\Phi(u)$ is given by

$$
\begin{aligned}
\mathscr{L}_{\Phi}(s) & =\int_{0}^{\infty} e^{-s u} \Phi(u) \mathrm{d} u \\
& =\frac{c-\mu_{1}}{c s+\left(\sigma^{2} / 2\right) s^{2}-\int_{0}^{\infty}\left(1-e^{-s x}\right) \nu(\mathrm{d} x)}, \quad s>0 .
\end{aligned}
$$

\section{Estimation of Survival Probability}

In our work, we assume that $\sigma$ and $v$ are unknown. In order to estimate $\Phi(u)$, we need to estimate the Laplace transform of $\Phi(u)$. Now, let us rewrite (7) as follows:

$$
\mathscr{L}_{\Phi}(s)=\frac{1-\rho}{(1 / c) \psi_{Y}(s)}, \quad s>0,
$$

where $\rho=\mu_{1} / c$ and $\psi_{Y}(s)=c s+(1 / 2) \sigma^{2} s^{2}-\int_{0}^{\infty}\left(1-e^{-s x}\right)$ $v(\mathrm{~d} x)$.

Suppose that a discrete sample $Y^{n}=\left\{Y_{t^{n}} \mid t_{i}^{n}=i h_{n} ; i=\right.$ $0,1,2, \ldots, n\}$ can be observed. Let $Z_{i}=Y_{t_{i}^{n}}-Y_{t^{n}}$ and $h_{n}=t_{i}^{n}-t_{i-1}^{n}>0$. Our interest is to estimate $\Phi(u)^{-1}$ by a sample $\left\{Z_{1}, Z_{2}, \ldots, Z_{n}\right\}$.

3.1. A Threshold Estimator of $\mathscr{L}_{\Phi}(s)$. In this part, we will construct an estimator of $\mathscr{L}_{\Phi}(s)$. If we can estimate $\rho$ and $\psi_{Y}(s)$ in $(8)$, the estimator of $\mathscr{L}_{\Phi}(s)$ will be given by a plugin device. By You and Cai [17], an estimator of $\psi_{Y}(s)$ has been given by

$$
\widehat{\psi}_{Y}(s)=\frac{1}{h_{n}}\left(\frac{1}{n} \sum_{k=1}^{n} e^{s Z_{k}}-1\right), \quad s>0 .
$$

Now, the following work is to estimate $\rho=\mu_{1} / c$. By Shimizu $[21,22]$, we introduce the filter which is defined by

$$
\begin{aligned}
& \mathscr{D}_{k}^{n}:=\left\{\omega \in \Omega ;\left(c h_{n}-Z_{k}\right)>h_{n}^{b}\right\}, \\
& \mathscr{C}_{k}^{n}:=\left\{\omega \in \Omega ;\left(c h_{n}-Z_{k}\right) \leq h_{n}^{b}\right\},
\end{aligned}
$$

where $b$ is a positive constant.

In Shimizu [21], the author assumed that $J=\left\{J_{t}, t \geq 0\right\}$ is a compound Poisson process. When $b \in(0,(1 / 2))$, it is easy to judge a jump occurred if $\left(c h_{n}-Z_{k}\right)>h_{n}^{b}$. As a result, $\left(c h_{n}-Z_{k}\right)$ can be an approximation of the jump size when $\left(c h_{n}-Z_{k}\right)>h_{n}^{b}$ and $h_{n} \longrightarrow 0$.

When $J=\left\{J_{t}, t \geq 0\right\}$ is a compound Poisson process, we can give the following estimator of $\rho$ :

$$
\widehat{\rho}=\frac{1}{c n h_{n}} \sum_{k=1}^{n}\left(c h_{n}-Z_{k}\right) \mathbf{I}_{\mathscr{D}_{k}^{n}}
$$

If $J=\left\{J_{t}, t \geq 0\right\}$ has possibly a infinite number of jumps in each finite time interval, we still choose $\hat{\rho}$ as an estimator of $\rho$.

Finally, by (8), (9), and (11), an estimator of $\mathscr{L}_{\Phi}(s)$ is given by

$$
\widehat{\mathscr{L}}_{\Phi}(s)=\frac{1-\hat{\rho}}{(1 / c) \widehat{\psi}_{Y}(s)}, \quad s>0
$$


3.2. A Regularized Laplace Inversion Technique. In [24], Chauveau et al. have given the following regularized Laplace inversion technique.

Definition 1. Let $m>0$ be a constant. The regularized Laplace inversion $L_{m}^{-1}: L^{2}(0, \infty) \longrightarrow L^{2}(0, \infty)$. is given by

$$
L_{m}^{-1} g(t)=\frac{1}{\pi^{2}} \int_{0}^{\infty} \int_{0}^{\infty} \Psi_{m}(y) y^{-1 / 2} e^{-t v y} g(v) \mathrm{d} v \mathrm{~d} y,
$$

for a function $g \in L^{2}(0, \infty)$ and $t \in(0, \infty)$, where

$$
\Psi_{m}(y)=\int_{0}^{a_{m}} \cosh (\pi x) \cos (x \log y) \mathrm{d} x,
$$

and $a_{m}=\pi^{-1} \cosh ^{-1}(\pi m)>0$.

By Definition 1, the regularized Laplace inversion technique is available for any $L^{2}(0, \infty)$ functions. According to the proof of Proposition 3.3 in You and Cai [17], we know that $\widehat{\mathscr{L}}_{\Phi}(s) \notin L^{2}(0, \infty)$. In order to apply Definition 1 , we must amend $\hat{\mathscr{L}}_{\Phi}(s)$.

Let $\Phi_{\theta}(u):=e^{-\theta u} \Phi(u)$ for arbitrary fixed $\theta>0$. Obviously, we have

$$
\mathscr{L}_{\Phi_{\theta}}(s)=\mathscr{L}_{\Phi}(s+\theta), \quad s>0
$$

Therefore, we will give an estimator of $\mathscr{L}_{\Phi_{\theta}}(s)$ as follows:

$$
\widehat{\mathscr{L}}_{\Phi_{\theta}}(s):=\widehat{\mathscr{L}}_{\Phi}(s+\theta)=\frac{1-\widehat{\rho}}{(1 / c) \widehat{\psi}_{Y}(s+\theta)}, \quad s>0 .
$$

By Definition 1, we can construct an estimator of $\Phi(u)$ as follows:

$$
\widehat{\Phi}_{m}(u)=e^{\theta u} \mathscr{L}_{m}^{-1} \widehat{\mathscr{L}}_{\Phi_{\theta}}(s), \quad u>0, \theta>0,
$$

for suitable $m>0$.

\section{Asymptotic Property of Estimators}

In this section, we will study the asymptotic property for those estimators which are proposed in Section 3. In [17], You and Cai have given the asymptotic normality and consistency of $\widehat{\psi}_{Y}(s)$. The following work will consider the asymptotic property of $\hat{\rho}, \widehat{\mathscr{L}}_{\Phi_{\theta}}(s)$, and $\widehat{\Phi}_{m}$.

If $J=\left\{J_{t}, t \geq 0\right\}$ is a compound Poisson process, we will give the following Theorems 1 and 2 .

Let $J_{t}=\sum_{j=1}^{N_{t}} \gamma_{j}$, where $N=\left\{N_{t}, t \geq 0\right\}$ is a Poisson process with constant intensity $\lambda$. The random variables $\gamma_{1}, \gamma_{2}, \gamma_{3}, \ldots$ are i.i.d. and independent of $N$. Let $\mu_{\gamma}=$ $E\left[\gamma_{1}\right]<\infty, \sigma_{\gamma}^{2}=E\left[\gamma_{1}^{2}\right]-\mu_{\gamma}^{2}<\infty$, and $\mathscr{L}_{\gamma}(s)=E\left[e^{-s \gamma_{1}}\right]$.

Theorem 1. Suppose that the net profit condition $c>\lambda \mu_{\gamma}$ hold. If $b \in(0,(1 / 2)), \theta>0, \lim _{n \longrightarrow \infty} h_{n}=0, \lim _{n \rightarrow \infty} n h_{n}=$ $\infty$, and $\lim _{n \longrightarrow \infty} n h_{n}^{1+\beta}=0$ for some $\beta \in(0,1)$, then for $n \longrightarrow \infty$, we have

$$
\begin{gathered}
\hat{\rho}-\rho \stackrel{P}{\longrightarrow} 0, \\
\sqrt{n h_{n}}(\hat{\rho}-\rho) \stackrel{D}{\longrightarrow} \mathcal{N}\left(0, \frac{1}{c^{2}} \lambda\left(\mu_{\gamma}^{2}+\sigma_{\gamma}^{2}\right)\right),
\end{gathered}
$$

$$
\begin{aligned}
& \sqrt{n h_{n}}\left(\hat{\mathscr{L}}_{\Phi_{\theta}}(s)-\mathscr{L}_{\Phi_{\theta}}(s)\right) \\
& \quad \stackrel{D}{\longrightarrow} \mathcal{N}\left(0, \frac{\lambda\left(\mu_{\gamma}^{2}+\sigma_{\gamma}^{2}+\sigma^{2}\right)}{\psi_{Y}^{2}(s+\theta)}+\frac{\mu_{\gamma}^{2} \psi_{Y}(2 s+2 \theta)}{\psi_{Y}^{4}(s+\theta)}\right) .
\end{aligned}
$$

Theorem 2. Suppose that $\Phi(u)$ has the first derivative $g(u)$ such that $g(u)$ is of the polynomial growth and the conditions of Theorem 1 are satisfied. Then, for $m=\sqrt{n h_{n} / \log \left(n h_{n}\right)}$, $u>0$ and any constant $B>0$, we have

$$
\left\|\widehat{\Phi}_{m}-\Phi\right\|_{B}^{2}=O_{P}\left(\frac{1}{\log \left(n h_{n}\right)}\right), \quad n \longrightarrow \infty \text {. }
$$

Now, we consider that the process $J$ contains lots of small jumps, i.e., an infinite number of jumps in each finite time interval. Theorem 3 will give the consistency for $\hat{\rho}$ and $\hat{\mathscr{L}}_{\Phi_{\theta}}(s)$.

Theorem 3. Suppose that the net profit condition $c>\mu_{1}$ hold. If $b \in(0,(1 / 4)), h_{n} \longrightarrow 0, n h_{n} \longrightarrow \infty$, and $n h_{n}^{1+\beta} \longrightarrow 0$ for some $\beta \in(0,1)$ as $n \longrightarrow \infty$, then

$$
\begin{array}{r}
\hat{\rho}-\rho \stackrel{P}{\longrightarrow} 0, \\
\hat{\mathscr{L}}_{\Phi_{\theta}}(s)-\mathscr{L}_{\Phi_{\theta}}(s) \stackrel{P}{\longrightarrow} 0 .
\end{array}
$$

Theorem 4. Suppose that $\Phi(u)$ has the first derivative $g(u)$ such that $g(u)$ is of the polynomial growth and the conditions of Theorem 3 are satisfied. Then, for $u>0, m>0$, and $B>0$, we have

$$
\left\|\widehat{\Phi}_{m}-\Phi\right\|_{B}^{2} \leq m^{2} o_{P}(1)+a_{m}, \quad n \longrightarrow \infty,
$$

where $a_{m}=O(1 / \log m)$ as $m \longrightarrow \infty$.

\section{Simulation}

In this section, we will give some simulation studies to show the performance of our estimator with finite samples. The work is based on MATLAB. We do not pretend to find an optimal threshold function for each considered model.

We assume that the Lévy measure is given by $\nu(\mathrm{d} x)=\lambda(1 / \eta) e^{-(1 / \eta) x} \mathrm{~d} x$, then $J$ is a compound Poisson process where the Poisson intensity is $\lambda$ and the individual claim sizes are exponentially distributed with mean $\eta$.

The survival probability is given by

$$
\begin{aligned}
\Phi(u)= & 1-\frac{r_{1}+(1 / \eta)+\left(2 \lambda \eta / \sigma^{2}\right)}{r_{1}-r_{2}} e^{r_{1} u} \\
& -\frac{r_{2}+(1 / \eta)+\left(2 \lambda \eta / \sigma^{2}\right)}{r_{2}-r_{1}} e^{r_{2} u},
\end{aligned}
$$

where $r_{2}<r_{1}<0$ are negative roots of the following equation

$$
\frac{1}{2} \sigma^{2} s+c-\frac{\lambda}{s+(1 / \eta)}=0 .
$$

We take $c=\lambda=10, \quad \eta=1 / 2, \quad \sigma=5, \quad b=1 / 4, \quad$ and $h_{n}=n^{-4 / 5}$. Then, $\mu_{1}=\lambda \eta=5$ and $\rho=\mu_{1} / c=0.5$. 


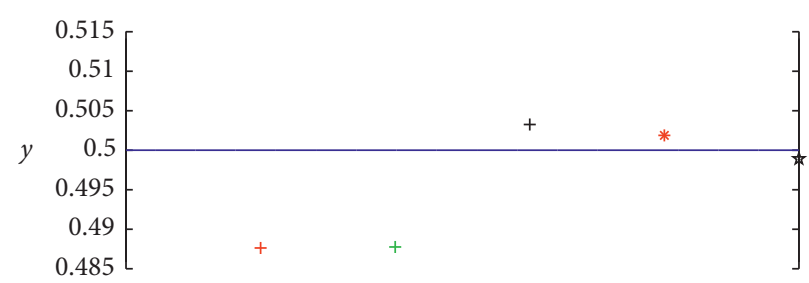

+ Estimator of $\rho$ with sample size $n=1000$

+ Estimator of $\rho$ with sample size $n=5000$

+ Estimator of $\rho$ with sample size $n=8000$

* Estimator of $\rho$ with sample size $n=10000$

\. Estimator of $\rho$ with sample size $n=30000$

The true value of $\rho$

Figure 1: $\hat{\rho}$ with sample sizes $n=1000,5000,8000,10000$, and 30000 .

TABLE 1: The data of $\hat{\rho}$ and its errors.

\begin{tabular}{lccr}
\hline Sample sizes & The data of $\hat{\rho}$ & The actual data of $\rho$ & Errors \\
\hline$n=1000$ & 0.4878 & 0.5 & 0.0122 \\
$n=5000$ & 0.4876 & 0.5 & 0.0124 \\
$n=8000$ & 0.5033 & 0.5 & 0.0033 \\
$n=10000$ & 0.5019 & 0.5 & 0.0019 \\
$n=30000$ & 0.4989 & 0.5 & 0.0011 \\
\hline
\end{tabular}

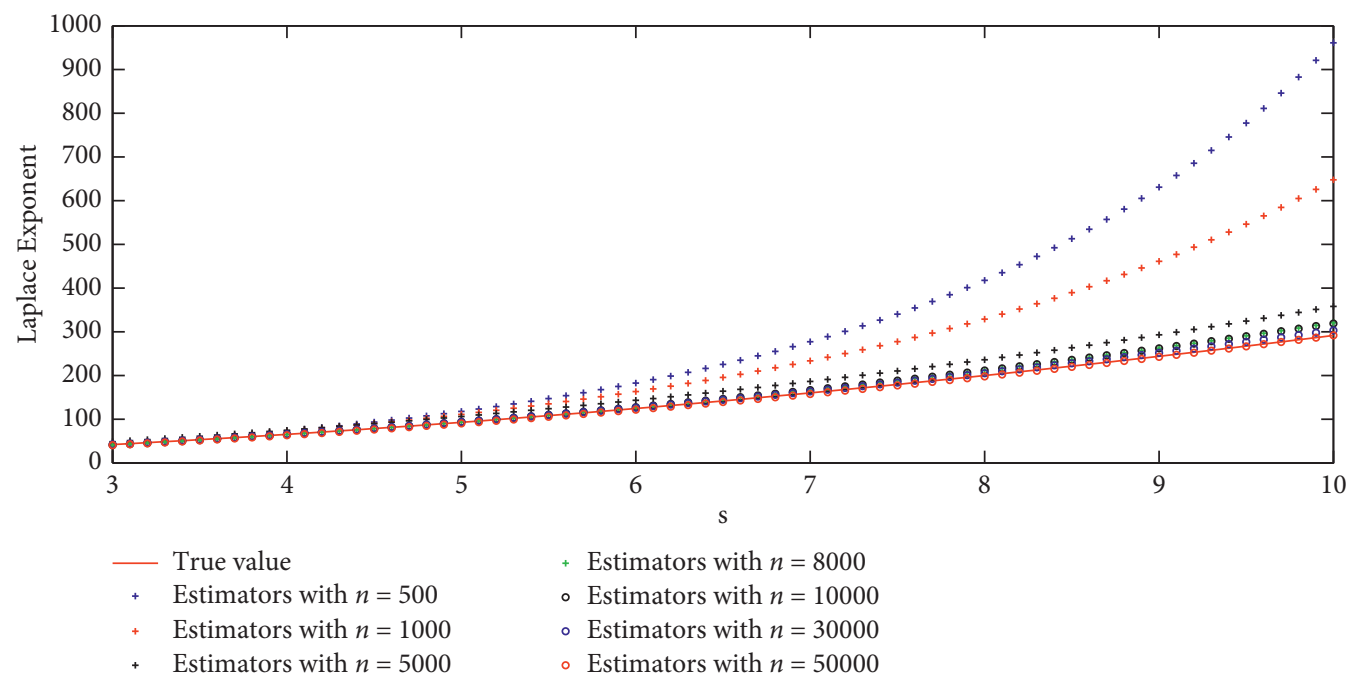

Figure 2: The estimator of $\psi_{Y}$ with sample sizes $n=500,1000,5000,8000,10000,30000$, and 50000 .

First of all, we consider $\widehat{\rho}$. In Figure 1, we plot the mean points with sample sizes $n=1000,5000,8000,10000$, and 30000 , which are computed based on 5000 simulation experiments.

In Table 1 , we give the data of $\hat{\rho}$ and its errors with sample sizes $n=1000,5000,8000,10000$, and 30000 .

Now, we consider the estimator of $\psi_{Y}$. In Figure 2, we plot the mean points with sample sizes $n=500,1000,5000$, $8000,10000,30000$, and 50000, which are computed based on 5000 simulation experiments.

Next, we consider the estimator of $\mathscr{L}_{\Phi_{\theta}}$ with $\theta=0.075$. In Figure 3, we plot the mean points with sample sizes $n=5000,10000,30000,50000$, and 80000 , which are computed based on 5000 simulation experiments.

By Figure 3, the estimator $\widehat{\mathscr{L}}_{\Phi_{\theta}}$ is very close to the actual data of $\mathscr{L}_{\Phi_{\theta}}$ as $n \geq 30000$. Thus, we will use the same method as of Cai et al. [18] and You and Cai [17] to simulate $\Phi(u)$. In order to improve computational efficiency, we define

$$
\Phi_{p}(u)=\left(e^{u \theta} / \pi^{2}\right) \int_{0}^{\infty} \int_{0}^{\infty} e^{-u s y}\left(\hat{\mathscr{L}}_{\Phi_{\theta}}(s)\right)_{n_{0}} \Psi_{p}(y) y^{-1 / 2} \mathrm{~d} s \mathrm{~d} y,
$$

where $\left(\hat{\mathscr{L}}_{\Phi_{\theta}}(s)\right)_{n_{0}}=1-\left(1 / c n_{0} h_{n_{0}}\right) \quad \sum_{k=1}^{n_{0}}\left(c h_{n_{0}}-Z_{k}\right) \mathbf{I}_{\mathscr{D}_{k}^{n_{0}}} /$ $\left(1 / c h_{n_{0}}\right)\left(\left(1 / n_{0}\right) \sum_{k=1}^{n_{0}} e^{s Z_{k}}-1\right)$, 


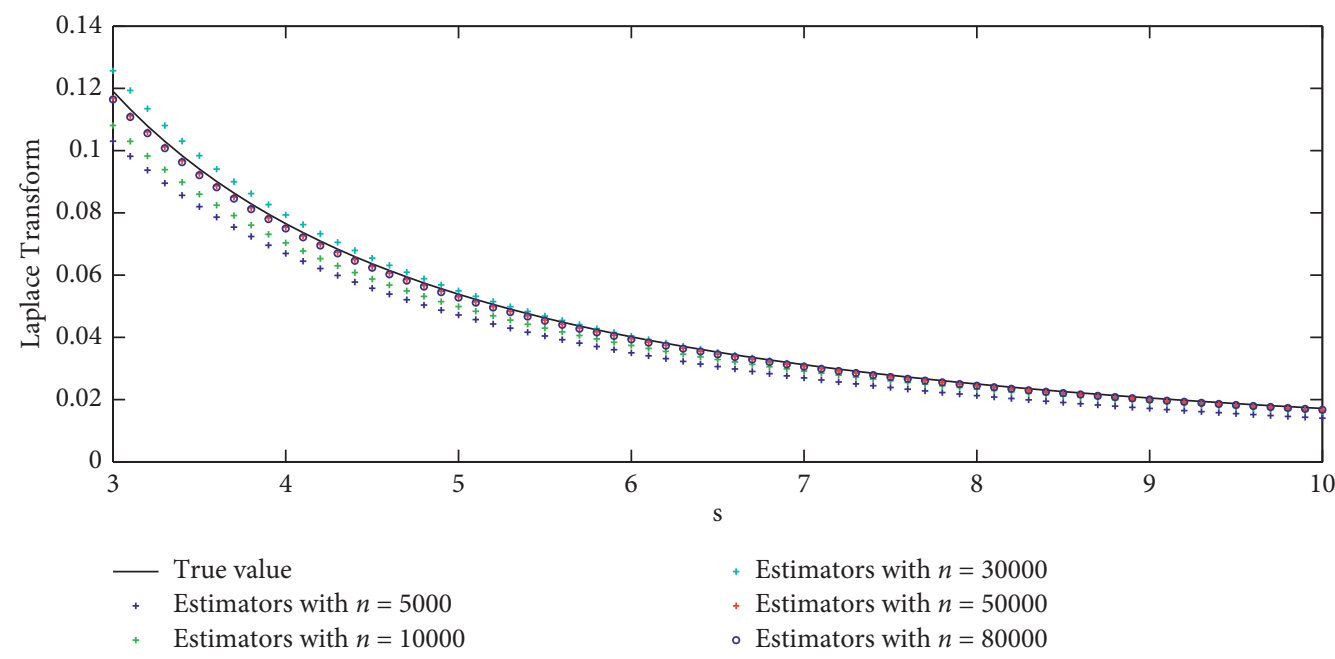

Figure 3: The estimator of $\mathscr{L}_{\Phi_{\theta}}$ with sample sizes $n=5000,10000,30000,50000$, and 80000 .

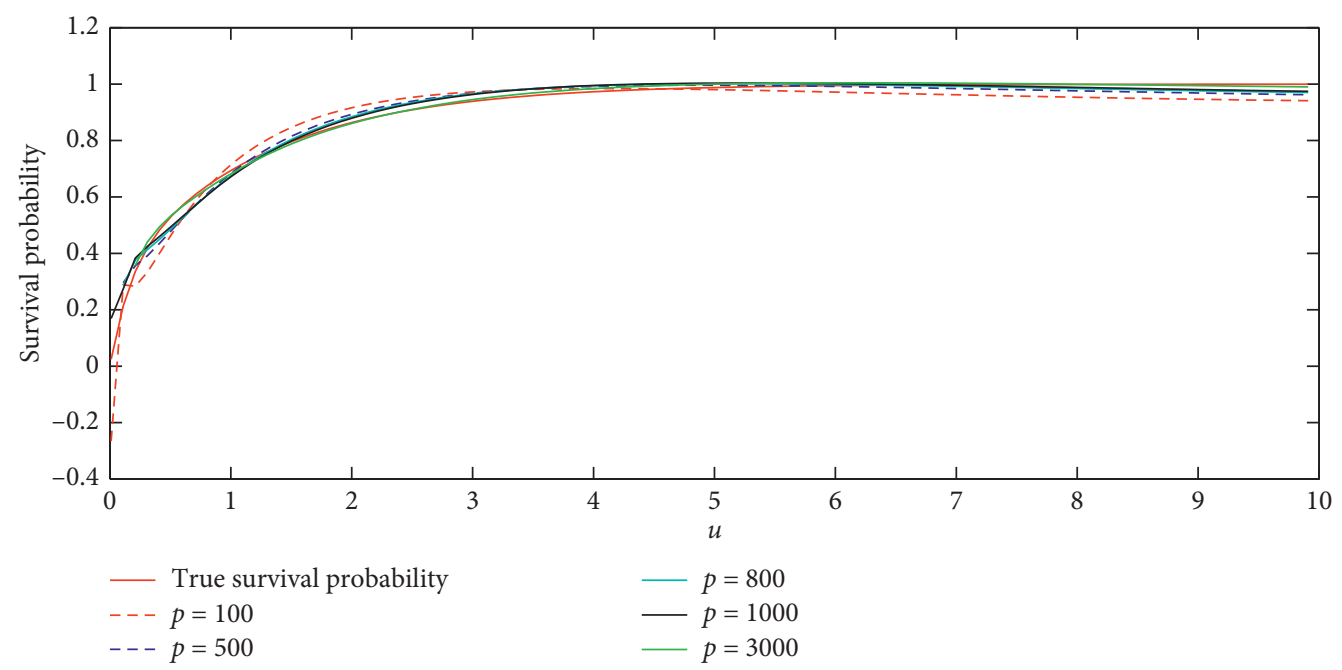

FIgURE 4: True curve and mean curves with sample size $n=30000$ and $p=100,500,800,1000$, and 3000.

$\Psi_{p}(y)=\int_{0}^{a_{p}} \cosh (\pi x) \cos (x \log (y)) \mathrm{d} x \quad$ and $\quad a_{p}=\pi^{-1}$ $\cosh ^{-1}(\pi p)>0$, and $n_{0}=30000$.

Now, we will show the performance of $\Phi_{p}(u)$.

In Figure 4, we plot the mean points with sample sizes $n=30000$ and $p=100,500,800,1000$, and 3000, which are computed based on 5000 simulation experiments.

\section{Conclusion}

In this paper, we use the threshold estimation technique and regularized Laplace inversion technique to construct an estimator of survival probability for a spectrally negative Lévy process. The rate of convergence for the estimator is a logarithmic rate. We adopt a method which is proposed in Cai et al. [18] to improve the speed in simulated calculation. The further work is to improve the speed of convergence for the estimator. We will combine the threshold estimation technique with the Fourier transform (inversion) technique, Fourier cosine series expansion method, and Laguerre series expansion method to construct an estimator of survival probability. These methods can be referred to Zhang and $\mathrm{Hu}[12,25]$, Zhang and $\mathrm{Su}[26,27], \mathrm{Yu}$ et al. [28], and so on. We hope some further studies will be performed for the risk model with the barrier or threshold dividend strategy. See, e.g., Peng et al. [29] and Yu et al. [30]. The Gerber-Shiu function and the aggregate dividends up to ruin will be estimated by some statistical methods.

\section{Appendix}

The proof of Theorem 1

Proof. First of all, we have 


$$
\begin{aligned}
\sqrt{n h_{n}}(\widehat{\rho}-\rho)= & \sqrt{n h_{n}}\left(\frac{1}{c n h_{n}} \sum_{k=1}^{n}\left(c h_{n}-Z_{k}\right) I_{\mathscr{D}_{k}^{n}}-\frac{\lambda \mu_{\gamma}}{c}\right) \\
= & \sqrt{n h_{n}}\left(\frac{1}{c n h_{n}} \sum_{k=1}^{n}\left(c h_{n}-Z_{k}\right) I_{\mathscr{D}_{k}^{n}}-\frac{1}{c n h_{n}} \sum_{k=1}^{n} \mu_{\gamma} I_{\left\{\Delta_{i}^{n} N \geq 2\right\}}+\frac{1}{c n h_{n}} \sum_{k=1}^{n} \mu_{\gamma} I_{\left\{\Delta_{i}^{n} N \geq 2\right\}}-\frac{\lambda \mu_{\gamma}}{c}\right) \\
= & \frac{1}{c \sqrt{n h_{n}}} \sum_{k=1}^{n}\left(c h_{n}-Z_{k}\right) I_{\mathscr{D}_{k}^{n} I_{\left\{\Delta_{i}^{n} N=0\right\}}}+\frac{1}{c \sqrt{n h_{n}}} \sum_{k=1}^{n}\left(\left(c h_{n}-Z_{k}\right) I_{\mathscr{D}_{k}^{n}}-\mu_{\gamma}\right) I_{\left\{\Delta_{i}^{n} N \geq 2\right\}} \\
& +\sqrt{n h_{n}}\left(\frac{1}{c n h_{n}} \sum_{k=1}^{n}\left(c h_{n}-Z_{k}\right) I_{\mathscr{D}_{k}^{n}} I_{\left\{\Delta_{k}^{n} N=1\right\}}-\frac{\lambda \mu_{\gamma}}{c}\right) \\
& +\sqrt{n h_{n}}\left(\frac{1}{c n h_{n}} \sum_{k=1}^{n} \mu_{\gamma} I_{\left\{\Delta_{k}^{n} N \geq 2\right\}}\right) .
\end{aligned}
$$

Let us now deal with the first term of (A.1),

$$
\begin{aligned}
& P\left(\frac{1}{c \sqrt{n h_{n}}} \sum_{k=1}^{n}\left(c h_{n}-Z_{k}\right) I_{\mathscr{D}_{k}^{n}} I_{\left\{\Delta_{i}^{n} N=0\right\}} \neq 0\right) \\
& \quad \leq P\left(\cup_{k=1}^{n}\left\{\left(c h_{n}-Z_{k}\right)>h_{n}^{b}, \Delta_{k}^{n} N=0\right\}\right) \\
& \quad \leq P\left(\sup _{k}\left(c h_{n}-Z_{k}\right) I_{\left\{\Delta_{k}^{n} N=0\right\}}>h_{n}^{b}\right) \\
& \quad \leq P\left(\sup _{k}\left|\sigma\left(W_{t_{k}^{n}}-W_{t_{k-1}^{n}}\right)\right|>h_{n}^{b}\right) \\
& \quad \leq \sum_{k} P\left(\left|\sigma\left(W_{t_{k}^{n}}-W_{t_{k-1}^{n}}\right)\right|>h_{n}^{b}\right) .
\end{aligned}
$$

By Proposition 3.2 and Corollary 3.3 in Mancini [19],

$$
\sum_{k} P\left(\left|\sigma\left(W_{t_{k}^{n}}-W_{t_{k-1}^{n}}\right)\right|>h_{n}^{b}\right) \leq n 2 e^{-h_{n}^{2 b} / 2 \sigma^{2} h_{n}}=2 n e^{-h_{n}^{(2 b-1)} / 2 \sigma^{2}} .
$$

Because of $b \in 0(0,(1 / 2)), \quad 2 n e^{-h_{n}^{(2 b-1)} / 2 \sigma^{2}} \longrightarrow 0$ as $n \longrightarrow \infty$.

Thus, the first term tends to zero in probability.

The second term of (A.1) tends to zero in probability, since

$$
\begin{aligned}
& P\left(\frac{1}{c \sqrt{n h_{n}}} \sum_{k=1}^{n}\left(\left(c h_{n}-Z_{k}\right) I_{D_{k}^{n}}-\mu\right) I_{\left\{\Delta_{i}^{n} N \geq 2\right\}} \neq 0\right) \\
& \quad \leq P\left(\cup_{k=1}^{n}\left\{\Delta_{i}^{n} N \geq 2\right\}\right) \\
& \quad \leq O\left(n h_{n}^{2}\right) .
\end{aligned}
$$

Therefore, we only need to compute the limit in distribution of the third term of (A.1).

By Mancini [19], we know $P \lim _{n \longrightarrow \infty} I_{\mathscr{D}_{k}^{n}}=$ $P \lim _{n \longrightarrow \infty} I_{\left\{\Delta_{k}^{n} N=1\right\}}$, then

$$
\begin{aligned}
& d \lim _{n \longrightarrow \infty} \sqrt{n h_{n}}\left(\frac{1}{c n h_{n}} \sum_{k=1}^{n}\left(c h_{n}-Z_{k}\right) I_{\mathscr{D}_{k}^{n}} I_{\left\{\Delta_{k}^{n} N=1\right\}}-\frac{\lambda \mu}{c}\right) \\
& \quad=d \lim _{n \longrightarrow \infty} \sqrt{n h_{n}}\left(\frac{1}{c n h_{n}} \sum_{k=1}^{n}\left[\gamma_{k}-\sigma\left(W_{t_{k}^{n}}-W_{t_{k-1}^{n}}\right)\right] I_{\left\{\Delta_{k}^{n} N=1\right\}}-\frac{\lambda \mu}{c}\right) .
\end{aligned}
$$

Because the random variables $\gamma, N$, and $W$ are independent to each other and $\gamma_{k}$ are i.i.d, we have (A.5) that tends to $\mathcal{N}\left(0,\left(\lambda\left(\mu_{\gamma}^{2}+\sigma_{\gamma}^{2}\right) / c^{2}\right)\right)$ in distribution as $n \longrightarrow \infty$. Thus, we can obtain (19). By (19) and Slutsky's Theorem, it is easy to obtain (18).

Next, by (7) and (16), we have

$$
\begin{aligned}
& \sqrt{n h_{n}}\left(\hat{\mathscr{L}}_{\Phi_{\theta}}(s)-\mathscr{L}_{\Phi_{\theta}}(s)\right) \\
& =\sqrt{n h_{n}}\left(\frac{\hat{\rho}}{(1 / c) \widehat{\psi}_{Y}(s+\theta)}-\frac{\rho}{(1 / c) \psi_{Y}(s+\theta)}\right) \\
& =\sqrt{n h_{n}} \frac{\hat{\rho}\left(\psi_{Y}(s+\theta)-\widehat{\psi}_{Y}(s+\theta)\right)+\widehat{\psi}_{Y}(s+\theta)(\widehat{\rho}-\rho)}{(1 / c) \widehat{\psi}_{Y}(s+\theta) \psi_{Y}(s+\theta)} .
\end{aligned}
$$

By Slutsky's Theorem, (11), (9), and (A.6), we have 


$$
\begin{aligned}
\sqrt{n h_{n}}\left(\hat{\mathscr{L}}_{\Phi_{\theta}}(s)-\mathscr{L}_{\Phi_{\theta}}(s)\right)= & \sqrt{n h_{n}} \frac{\rho\left(\psi_{Y}(s+\theta)-\widehat{\psi}_{Y}(s+\theta)\right)+\psi_{Y}(s+\theta)(\hat{\rho}-\rho)}{(1 / c)\left(\psi_{Y}(s+\theta)\right)^{2}}+o_{P}(1) \\
= & \sqrt{n h_{n}} \frac{\psi_{Y}(s+\theta)\left(\left(\sum_{k=1}^{n}\left(c h_{n}-Z_{k}\right) I_{D_{k}^{n}} / c n h_{n}\right)-\rho\right)}{(1 / c)\left(\psi_{Y}(s+\theta)\right)^{2}} \\
= & -\sqrt{n h_{n}} \frac{\rho\left(\left((1 / n) \sum_{k=1}^{n} e^{\left.\left.(s+\theta) Z_{k}-1 / h_{n}\right)-\psi_{Y}(s+\theta)\right)}\right.\right.}{(1 / c)\left(\psi_{Y}(s+\theta)\right)^{2}}+o_{P}(1) \\
= & \sqrt{n h_{n}} \sum_{k=1}^{n} \frac{\psi_{Y}(s+\theta)\left(\left(c h_{n}-Z_{k}\right) I_{D_{k}^{n}} / c n h_{n}\right)-\rho\left(e^{(s+\theta) Z_{k}}-1\right) / n h_{n}}{(1 / c)\left(\psi_{Y}(s+\theta)\right)^{2}}+o_{P}(1) \\
= & \frac{1}{\sqrt{n h_{n}}} \sum_{k=1}^{n} \frac{\psi_{Y}(s+\theta)\left[\left(c h_{n}-Z_{k}\right) I_{\mathscr{D}_{k}^{n}}\right]-\rho c\left[e^{\left.(s+\theta) Z_{k}-1\right]}\right.}{\left(\psi_{Y}(s+\theta)\right)^{2}} \\
& +o_{P}(1) .
\end{aligned}
$$

By $P \lim _{n \longrightarrow \infty} I_{\mathscr{D}_{k}^{n}}=P \lim _{n \longrightarrow \infty} I_{\left\{\Delta_{k}^{n} N=1\right\}}$,

$$
\begin{aligned}
& d \lim _{n \rightarrow \infty} \frac{1}{\sqrt{n h_{n}}} \sum_{k=1}^{n} \frac{\psi_{Y}(s+\theta)\left[\left(c h_{n}-Z_{k}\right) I_{\mathscr{D}_{k}^{n}}\right]-\rho c\left[e^{(s+\theta) Z_{k}}-1\right]}{\left(\psi_{Y}(s+\theta)\right)^{2}} \\
& \quad=d \lim _{n \longrightarrow \infty} \frac{1}{\sqrt{n h_{n}}} \sum_{k=1}^{n} \frac{\psi_{Y}(s+\theta)\left[\left(c h_{n}-Z_{k}\right) I_{\Delta_{k}^{n} N=1}\right]-\rho c\left[e^{(s+\theta) Z_{k}}-1\right]}{\left(\psi_{Y}(s+\theta)\right)^{2}} . \\
& \text { property of } \gamma, N, \quad W, \text { and } \quad\left\|\mathscr{L}_{n}^{-1} \mathscr{L}_{f}-f\right\|=O\left((\log n)^{-1 / 2}\right), \quad(n \longrightarrow \infty) .
\end{aligned}
$$

By the independent property of $\gamma, N, W$, and

$\gamma_{1}, \gamma_{2}, \gamma_{3}, \ldots, \gamma_{n}$

$$
\begin{aligned}
& \sqrt{n h_{n}}\left(\hat{\mathscr{L}}_{\Phi_{\theta}}(s)-\mathscr{L}_{\Phi_{\theta}}(s)\right) \\
& \stackrel{D}{\longrightarrow} \mathcal{N}\left(0, \frac{\lambda\left(\mu_{\gamma}^{2}+\sigma_{\gamma}^{2}+\sigma^{2}\right)}{\psi_{Y}^{2}(s+\theta)}+\frac{\mu_{\gamma}^{2} \psi_{Y}(2 s+2 \theta)}{\psi_{Y}^{4}(s+\theta)}\right) .
\end{aligned}
$$

In order to prove Theorem 2, we need the following Lemma 1 (see Theorem 3.2 in [24]).

Lemma 1. Suppose that for a function $f \in L^{2}(0, \infty)$ with the derivative $f^{\prime}, \int_{0}^{\infty}\left[t\left(t^{1 / 2} f(t)\right)^{\prime}\right]^{2} t^{-1} d t<\infty$, then
The proof of Theorem 2

Proof. Using (17),

$$
\begin{aligned}
\left\|\widehat{\Phi}_{m}-\Phi\right\|_{B}^{2} & \leq e^{2 \theta B}\left\|\widehat{\Phi}_{m} e^{-\theta u}-\Phi_{\theta}\right\|_{B}^{2} \\
& \leq 2 e^{2 \theta B}\left\{\left\|\mathscr{L}_{m}^{-1} \widehat{\mathscr{L}}_{\Phi_{\theta}}-\mathscr{L}_{m}^{-1} \mathscr{L}_{\Phi_{\theta}}\right\|^{2}+\left\|\mathscr{L}_{m}^{-1} \mathscr{L}_{\Phi_{\theta}}-\Phi_{\theta}\right\|^{2}\right\} .
\end{aligned}
$$

Let $\Phi_{\theta}^{\prime}=g_{\theta}$. Because $g(u)$ satisfies the polynomial growth,

$$
\begin{aligned}
\int_{0}^{\infty}\left[x\left(\sqrt{x} \Phi_{\theta}(x)\right)^{\prime}\right]^{2}(1 / x) \mathrm{d} x & \leq \int_{0}^{\infty} \Phi_{\theta}^{2}(x) \mathrm{d} x+\int_{0}^{\infty} x^{2} g_{\theta}^{2}(x) \mathrm{d} x \\
& =\left\|\Phi_{\theta}\right\|^{2}+\int_{0}^{\infty} x^{2}\left[g(x) e^{-\theta x}-\theta \Phi(x) e^{-\theta x}\right]^{2} \mathrm{~d} x \\
& \leq\left\|\Phi_{\theta}\right\|^{2}+\int_{0}^{\infty} x^{2} g^{2}(x) e^{-2 \theta x} \mathrm{~d} x+\int_{0}^{\infty} x^{2} \Phi^{2}(x) e^{-2 \theta x} \mathrm{~d} x \\
& \leq\left\|\Phi_{\theta}\right\|^{2}+\int_{0}^{\infty} x^{2} e^{-2 \theta x}\left(M^{2}+1\right) \mathrm{d} x \\
& <\infty .
\end{aligned}
$$


By Lemma 1,

By (7), (12), (15), and (16), we have

$$
\left\|\mathscr{L}_{m}^{-1} \mathscr{L}_{\Phi_{\theta}}-\Phi_{\theta}\right\|^{2}=O\left(\frac{1}{\log m}\right), \quad m \longrightarrow \infty .
$$

$$
\left\|\widehat{\mathscr{L}}_{\Phi_{\theta}}-\mathscr{L}_{\Phi_{\theta}}\right\|^{2}=\int_{0}^{\infty}\left(\frac{(\rho-1)\left((1 / c) \widehat{\psi}_{Y}(s+\theta)-(1 / c) \psi_{Y}(s+\theta)\right)}{(1 / c) \widehat{\psi}_{Y}(s+\theta)(1 / c) \psi_{Y}(s+\theta)}+\frac{(\rho-\hat{\rho})}{(1 / c) \widehat{\psi}_{Y}(s+\theta)}\right)^{2} \mathrm{~d} s .
$$

Using the proof of Propositions 2.3 and 2.4 in You and Cai [17], the right-hand side in (A.14) is bounded by

$$
\frac{2}{(1-\widehat{\rho})^{2}} I_{1}+\frac{2(\widehat{\rho}-\rho)^{2}}{(1-\widehat{\rho})^{2}} I_{2}
$$

where

$$
\begin{aligned}
& I_{1}=\int_{0}^{\infty} \frac{1}{c^{2}} \frac{\left(\widehat{\psi}_{Y}(s+\theta)-\psi_{Y}(s+\theta)\right)^{2}}{(s+\theta)^{4}} \mathrm{~d} s, \\
& I_{2}=\int_{0}^{\infty} \frac{1}{(s+\theta)^{2}} \mathrm{~d} s<\infty .
\end{aligned}
$$

By Theorem 1, it follows that

$$
\begin{aligned}
I_{1} & =O_{P}\left(\frac{1}{n h_{n}}\right), \\
\frac{2}{(1-\hat{\rho})^{2}} & =O_{P}(1),
\end{aligned}
$$

$$
\begin{aligned}
\widehat{\rho}-\rho= & \frac{\sum_{k=1}^{n}\left(c h_{n}-Z_{k}\right) I_{\mathscr{D}_{k}^{n}}}{c n h_{n}}-\frac{\mu_{1}}{c} \\
\leq & \frac{\sum_{k=1}^{n}\left(\Delta_{k}^{n} J_{1}-\sigma\left(W_{t_{k}^{n}}-W_{t_{k-1}^{n}}\right)\right) I\left\{\left|\Delta_{k}^{n} J_{1}-\sigma\left(W_{t_{k}^{n}-W_{t_{k-1}^{n}}}\right)\right|>(1 / 2) h_{n}^{b}\right\}}{c n h_{n}}-\frac{E\left[J_{11}\right]}{c} \\
& +\frac{\sum_{k=1}^{n}\left(\Delta_{k}^{n} J_{1}-\sigma\left(W_{t_{k}^{n}}-W_{t_{k-1}^{n}}\right)\right)\left(I_{\mathscr{D}_{k}^{n}}-I\left\{\left|\left(\Delta_{k}^{n} J_{1}-\sigma W_{t_{k}^{n}-W_{t_{k-1}^{n}}}\right)\right|>(1 / 2) h_{n}^{b}\right\}\right)}{c n h_{n}} \\
& +\frac{\sum_{k=1}^{n} \Delta_{k}^{n} J_{2}}{c n h_{n}}-\frac{E\left[J_{21}\right]}{c} \\
& +\frac{\sum_{k=1}^{n} \Delta_{k}^{n} J_{2} I_{\mathscr{C}_{k}^{n}}}{c n h_{n}} .
\end{aligned}
$$

Let us consider the second term of (A.22), 


$$
\begin{aligned}
& \frac{\sum_{k=1}^{n}\left(\Delta_{k}^{n} J_{1}-\sigma\left(W_{t_{k}^{n}}-W_{t_{k-1}^{n}}\right)\right) I_{\mathscr{D}_{k}^{n}}}{c n h_{n}} \\
& -\frac{\sum_{k=1}^{n}\left(\Delta_{k}^{n} J_{1}-\sigma\left(W_{t_{k}^{n}}-W_{t_{k-1}^{n}}\right)\right) I\left\{\left|\Delta_{k}^{n} J_{1}-\sigma\left(W_{t_{k}^{n}-W_{t_{k-1}^{n}}}\right)\right|>(1 / 2) h_{n}^{b}\right\}}{c_{n h}} \\
& \leq \frac{\sum_{k=1}^{n}\left(\Delta_{k}^{n} J_{1}-\sigma\left(W_{t_{k}^{n}}-W_{t_{k-1}^{n}}\right)\right) I\left\{\left|\Delta_{k}^{n} J_{1}-\sigma\left(W_{t_{k}^{n}-W_{t_{k-1}^{n}}}\right)\right|+\left|\Delta_{k}^{n} J_{2}\right|>h_{n}^{b}\right\}}{c n h_{n}} \\
& -\frac{\sum_{k=1}^{n}\left(\Delta_{k}^{n} J_{1}-\sigma\left(W_{t_{k}^{n}}-W_{t_{k-1}^{n}}\right)\right) I\left\{\left|\Delta_{k}^{n} J_{1}-\sigma\left(W_{t_{k}^{n}-W_{t_{k-1}^{n}}}\right)\right|>(1 / 2) h_{n}^{b}\right\}}{\operatorname{cnh}_{n}} \\
& \leq \frac{\sum_{k=1}^{n}\left(\Delta_{k}^{n} J_{1}-\sigma\left(W_{t_{k}^{n}}-W_{t_{k-1}^{n}}\right)\right) I_{\left\{\left|\Delta_{k}^{n} J_{2}\right|>\left(h_{n}^{b} / 2\right)\right\}}}{c n h_{n}} \\
& \leq \frac{\sum_{k=1}^{n}\left|\Delta_{k}^{n} J_{1}\right| I_{\left\{\left|\Delta_{k}^{n} J_{2}\right|>\left(h_{n}^{b} / 2\right)\right\}}}{\operatorname{cnh}_{n}}+\frac{\sum_{k=1}^{n}\left|\sigma\left(W_{t_{k}^{n}}-W_{t_{k-1}^{n}}\right)\right| I_{\left\{\left|\Delta_{k}^{n} J_{2}\right|>\left(h_{n}^{b} / 2\right)\right\}}}{c_{n} h_{n}}
\end{aligned}
$$

By the Paul Lévy law for the modulus of continuity of Brownian motion's path (Vondraček and Shreve [31], p.114, Theorem 9.25) implies that

$$
\lim _{h_{n} \longrightarrow 0} \sup _{k \in\{1,2, \ldots, n\}} \frac{\left|\left(W_{t_{k}^{n}}-W_{t_{k-1}^{n}}\right)\right|}{\sqrt{h_{n} \ln \left(1 / h_{n}\right)}} \leq 1 \text {, a.s. }
$$

We have

$$
\begin{aligned}
& \frac{\sum_{k=1}^{n} \mid \sigma\left(W_{t_{k}^{n}}-W_{t_{k-1}^{n}}\right) I_{\left\{\left|\Delta_{k}^{n} J_{2}\right|>\left(h_{n}^{b} / 2\right)\right\} \mid}}{c n h_{n}} \\
& \quad \leq \sqrt{h_{n} \ln \left(\frac{1}{h_{n}}\right)} \frac{\sum_{k=1}^{n} I_{\left\{\left|\Delta_{k}^{n} J_{2}\right|>\left(h_{n}^{b} / 2\right)\right\}}}{\operatorname{cnh}_{n}} \stackrel{P}{\longrightarrow} 0,
\end{aligned}
$$

since

$$
\begin{aligned}
\sqrt{h_{n} \ln \left(\frac{1}{h_{n}}\right)} \frac{1}{h_{n}} P\left(\left(\Delta_{k}^{n} J_{2}\right)^{2}>\frac{h_{n}^{2 b}}{4}\right) & \leq \sqrt{h_{n} \ln \left(\frac{1}{h_{n}}\right)} \frac{1}{h_{n}} \frac{E\left[\left(\Delta_{k}^{n} J_{2}\right)^{2}\right]}{h_{n}^{2 b} / 4} \\
& =4 \sigma^{2} \frac{\sqrt{h_{n} \ln \left(1 / h_{n}\right)}}{h_{n}^{2 b}} \longrightarrow 0,
\end{aligned}
$$

as $n \longrightarrow \infty$ and $b \in(0,(1 / 4))$.

Moreover,

$$
\begin{aligned}
& P\left(\frac{\sum_{k=1}^{n}\left|\Delta_{k}^{n} J_{1}\right| I_{\left\{\left|\Delta_{k}^{n} J_{2}\right|>\left(h_{n}^{b} / 2\right)\right\}}}{c n h_{n}} \neq 0\right) \\
& \quad=P\left(\frac{\sum_{k=1}^{n}\left|\sum_{j=1}^{\Delta_{k} N} \gamma_{j}\right| I_{\left\{\left|\Delta_{k}^{n} J_{2}\right|>\left(h_{n}^{b} / 2\right)\right\}}}{c n h_{n}} \neq 0\right) \\
& \quad \leq P\left(\bigcup_{k=1}^{n}\left\{\Delta_{k} N \neq 0,\left(\Delta_{k}^{n} J_{2}\right)^{2}>h_{n}^{2 b}\right\}\right) \\
& \quad \leq n P\left(\Delta_{1} N \neq 0\right) \frac{E\left[\left(\Delta_{k}^{n} J_{2}\right)^{2}\right]}{h_{n}^{2 b}} \\
& \quad=n O\left(h_{n}\right) \frac{\sigma^{2}(1) h_{n}}{h_{n}^{2 b}} 0,
\end{aligned}
$$

since for $\forall \beta>0, n h_{n}^{1+\beta} \longrightarrow 0$.

Now, let us consider the last term of the right-hand side of (A.22): 
$\frac{\sum_{k=1}^{n} \Delta_{k}^{n} J_{2} I_{\mathscr{C}_{k}^{n}}}{c n h_{n}}=\frac{\left.\sum_{k=1}^{n} \Delta_{k}^{n} J_{2} I_{\{}\left|c h_{n}-Z_{k}\right| \leq h_{n}^{h}||_{k}^{n} J_{2} \mid>2 h_{n}^{b}\right\}}{c n h_{n}}$

$$
\begin{aligned}
& +\frac{\sum_{k=1}^{n} \Delta_{k}^{n} J_{2} I_{\left\{\left|c h_{n}-Z_{k}\right| \leq h_{n}^{b}\left|\Delta_{k}^{n} J_{2}\right| \leq 2 h_{n}^{b}\right\}}}{c n h_{n}} \\
\leq & \frac{\sum_{k=1}^{n} \Delta_{k}^{n} J_{2} I\left\{\left|\Delta_{k}^{n} J_{1}+\Delta_{k}^{n} J_{2}-\sigma\left(W_{t_{k}^{n}}-W_{t_{k-1}^{n}}\right)\right| \leq h_{n}^{b}\left|\Delta_{k}^{n} J_{2}\right|>2 h_{n}^{b}\right\}}{c n h_{n}}
\end{aligned}
$$$$
+\frac{\sum_{k=1}^{n} \Delta_{k}^{n} J_{2} I\left\{\left|\Delta_{k}^{n} J_{1}+\Delta_{k}^{n} J_{2}-\sigma\left(W_{t_{k}^{n}}-W_{t_{k-1}^{n}}\right)\right| \leq h_{n}^{b}\left|\Delta_{k}^{n} J_{2}\right| \leq 2 h_{n}^{b}\right\}}{c n h_{n}} .
$$

In fact, if

$$
\left|\Delta_{k}^{n} J_{1}+\Delta_{k}^{n} J_{2}-\sigma\left(W_{t_{k}^{n}}-W_{t_{k-1}^{n}}\right)\right| \leq h_{n}^{b},\left|\Delta_{k}^{n} J_{2}\right|>2 h_{n}^{b},
$$

then

$$
\begin{aligned}
2 h_{n}^{b}-\left|\Delta_{k}^{n} J_{1}-\sigma\left(W_{t_{k}^{n}}-W_{t_{k-1}^{n}}\right)\right| & <\left|\Delta_{k}^{n} J_{2}\right|-\left|\Delta_{k}^{n} J_{1}-\sigma\left(W_{t_{k}^{n}}-W_{t_{k-1}^{n}}\right)\right| \\
& \leq\left|\Delta_{k}^{n} J_{1}+\Delta_{k}^{n} J_{2}-\sigma\left(W_{t_{k}^{n}}-W_{t_{k-1}^{n}}\right)\right| \\
& \leq h_{n}^{b},
\end{aligned}
$$

so that

$$
\left|\Delta_{k}^{n} J_{1}\right|>\frac{1}{2} h_{n}^{b} \text { or }\left|\sigma\left(W_{t_{k}^{n}}-W_{t_{k-1}^{n}}\right)\right|>\frac{1}{2} h_{n}^{b}
$$

$\begin{aligned} & \text { since a.s., for small } h_{n}, I_{\{=1,2, . ., n \text {, then }} \\ & \left\{\mid \sigma\left(W_{\left.t_{k}^{n}-W_{t_{k-1}^{n}}\right) \mid>(1 / 2) h_{n}^{b}}\right\}\right.\end{aligned}=0$, for all

$$
\begin{aligned}
& P\left(\frac{\sum_{k=1}^{n}\left|\Delta_{k}^{n} J_{2}\right| I\left\{\left|\Delta_{k}^{n} J_{1}+\Delta_{k}^{n} J_{2}-\sigma\left(W_{t_{k}^{n}-W_{t_{k-1}^{n}}}\right)\right| \leq h_{n}^{b}\left|\Delta_{k}^{n} J_{2}\right|>2 h_{n}^{b}\right\}}{c n h_{n}} \neq 0\right) \\
& \leq P\left(\bigcup_{k=1}^{n}\left\{\left|\Delta_{k}^{n} J_{2}\right|>2 h_{n}^{b}, \Delta_{k}^{n} N \neq 0\right\}\right) \\
& \leq n P\left(\Delta_{k}^{n} N \neq 0\right) \frac{E\left[\left(\Delta_{k}^{n} J_{2}\right)^{2}\right]}{4 h_{n}^{2 b}} \\
& =n O\left(h_{n}^{2}\right) \frac{4 \sigma^{2}(1)}{h_{n}^{2 b}} \longrightarrow 0,
\end{aligned}
$$

as $n \longrightarrow \infty$, since $b \in(0,(1 / 4))$.

Now, let us consider the term:

$$
\begin{gathered}
P \lim _{h_{n} \longrightarrow 0} \frac{\sum_{k=1}^{n} \Delta_{k}^{n} J_{2} I\left\{\left|\Delta_{k}^{n} J_{1}+\Delta_{k}^{n} J_{2}-\sigma\left(W_{t_{k}^{n}-W_{t_{k-1}^{n}}}\right)\right| \leq h_{n}^{b}\left|\Delta_{k}^{n} J_{2}\right| \leq 2 h_{n}^{b}\right\}}{c n h_{n}} \\
\quad \leq P \lim _{h_{n}} \longrightarrow 0 \frac{\sum_{k=1}^{n} \Delta_{k}^{n} J_{2} I_{\left\{\left|\Delta_{k}^{n} J_{2}\right| \leq 2 h_{n}^{b}\right\}}}{c n h_{n}} .
\end{gathered}
$$

Because $Y_{t}$ is a spectrally negative Lévy process, for any $\delta>0$, we have

$$
\begin{aligned}
0 & \leq \frac{\sum_{k=1}^{n} \Delta_{k}^{n} J_{2} I_{\left\{\left|\Delta_{k}^{n} J_{2}\right| \leq 2 h_{n}^{b}\right\}}}{c n h_{n}} \\
& \leq \frac{\sum_{k=1}^{n} \int_{t_{k-1}^{n}}^{t_{k}^{n}}\left[\int_{|x| \leq \delta+2 h_{n}^{b}} x[\mu(\mathrm{d} s, \mathrm{~d} x)-\nu(\mathrm{d} x) \mathrm{d} s]-h_{n} \int_{\delta+2 h_{n}^{b}<x \leq 1} x \nu(\mathrm{d} x)\right]}{c n h_{n}} \\
& \leq \frac{\sum_{k=1}^{n} \int_{t_{k-1}^{n}}^{t^{n}} \int_{|x| \leq \delta+2 h_{n}^{b}} x \mu(\mathrm{d} s, \mathrm{~d} x)}{c n h_{n}},
\end{aligned}
$$

since $\delta$ is arbitrary, we can conclude that $P \lim _{n \longrightarrow \infty}$ $\left(\sum_{k=1}^{n} \Delta_{k}^{n} J_{2} I_{\left\{\left|\Delta_{k}^{n} J_{2}\right| \leq 2 h_{n}^{b}\right\}} / c n h_{n}\right)=0$, as $E\left[\sum_{k=1}^{n} \int_{t_{k-1}^{n}}^{t_{k}^{n}} \int_{|x| \leq \delta+2 h_{n}^{b}}\right.$ $\left.x \mu(d s, d x) / c n h_{n}\right]=E\left[\int_{0}^{h_{n}} \int_{|x| \leq \delta+2 h_{n}} x \mu(\mathrm{d} s, \mathrm{~d} x)\right] / c h_{n}=(1 / c)$ $\int_{|x| \leq \delta+2 h_{n}^{b}} x v(\mathrm{~d} x) \longrightarrow 0, \delta \longrightarrow 0, n \longrightarrow \infty$.

By the law of large number, we know that the first term and thirst term of (A.22) are convergence to zero in probability.

By (7), (16), (22), Theorem 1, and continuous mapping theorem, it is easy to obtain (23).

Therefore, we obtain the result of Theorem 3.
The proof of Theorem 4

Proof. By (17), we have

$$
\begin{aligned}
\left\|\widehat{\Phi}_{m}-\Phi\right\|_{B}^{2} & \leq e^{2 \theta B}\left\|\widehat{\Phi}_{m} e^{-\theta u}-\Phi_{\theta}\right\|_{B}^{2} \\
& \leq 2 e^{2 \theta B}\left\{\left\|\mathscr{L}_{m}^{-1} \hat{\mathscr{L}}_{\Phi_{\theta}}-\mathscr{L}_{m}^{-1} \mathscr{L}_{\Phi_{\theta}}\right\|^{2}+\left\|\mathscr{L}_{m}^{-1} \mathscr{L}_{\Phi_{\theta}}-\Phi_{\theta}\right\|^{2}\right\} .
\end{aligned}
$$

By the proof of Theorem 2, we may conclude that 


$$
\begin{aligned}
a_{m}= & \left\|\mathscr{L}_{m}^{-1} \mathscr{L}_{\Phi_{\theta}}-\Phi_{\theta}\right\|^{2}=O\left(\frac{1}{\log m}\right), \quad m \longrightarrow \infty \\
\left\|\widehat{\mathscr{L}}_{\Phi_{\theta}}-\mathscr{L}_{\Phi_{\theta}}\right\|^{2} & \\
= & \int_{0}^{\infty}\left(\frac{(\rho-1)\left((1 / c) \widehat{\psi}_{Y}(s+\theta)-(1 / c) \psi_{Y}(s+\theta)\right)}{(1 / c) \widehat{\psi}_{Y}(s+\theta)(1 / c) \psi_{Y}(s+\theta)}\right. \\
& \left.+\frac{(\rho-\widehat{\rho})}{(1 / c) \widehat{\psi}_{Y}(s+\theta)}\right)^{2} \mathrm{~d} s \\
\leq & \frac{2}{(1-\widehat{\rho})^{2}} I_{1}+\frac{2(\widehat{\rho}-\rho)^{2}}{(1-\widehat{\rho})^{2}} I_{2},
\end{aligned}
$$

where

$$
\begin{aligned}
& I_{1}=\int_{0}^{\infty} \frac{1}{c^{2}} \frac{\left(\widehat{\psi}_{Y}(s+\theta)-\psi_{Y}(s+\theta)\right)^{2}}{(s+\theta)^{4}} \mathrm{~d} s \\
& I_{2}=\int_{0}^{\infty} \frac{1}{(s+\theta)^{2}} \mathrm{~d} s<\infty .
\end{aligned}
$$

By Theorem 3 and continuous mapping theorem, it follows that

$$
\left\|\widehat{\mathscr{L}}_{\Phi_{\theta}}-L_{\Phi_{\theta}}\right\|^{2}=o_{P}(1), \quad n \longrightarrow \infty .
$$

Combining $L_{m}^{-1} \leq \sqrt{\pi} m$ in Chauveau et al. [24], (A.36), and (A.39), we have

$$
\left\|\widehat{\Phi}_{m}-\Phi\right\|_{B}^{2} \leq m^{2} o_{P}(1)+a_{m}
$$

as $n \longrightarrow \infty$.

\section{Data Availability}

The data used to support the findings of this study are available from the corresponding author upon request.

\section{Conflicts of Interest}

The authors declare that they have no conflicts of interest.

\section{Acknowledgments}

The authors were supported by the Postdoctoral Science Foundation of China (Grant no. 2018M642634) and the National Natural Science Foundation of China (Grant no. 11571198).

\section{References}

[1] S. Asmussen and H. Albrecher, Ruin Probabilities, World Scientific, Singapore, 2nd edition, 2010.

[2] F. Dufresne and H. U. Gerber, "Risk theory for the compound Poisson process that is perturbed by diffusion," Insurance: Mathematics and Economics, vol. 10, no. 1, pp. 51-59, 1991.

[3] N. Veraverbeke, "Asymptotic estimates for the probability of ruin in a Poisson model with diffusion," Insurance: Mathematics and Economics, vol. 13, no. 1, pp. 57-62, 1993.
[4] V. Dermitzakis and K. Politis, "Asymptotics for the moments of the time to ruin for the compound Poisson model perturbed by diffusion," Methodology and Computing in Applied Probability, vol. 13, no. 4, pp. 749-761, 2011.

[5] S. Li, C. S. Zhang, and R. Wu, "Ruin estimates of diffusion models under constant interest rate," Chinese Journal of Applied Probability and Statisties, vol. 19, no. 1, pp. 79-84, 2003.

[6] K. Croux and N. Veraverbeke, "Nonparametric estimators for the probability of ruin," Insurance: Mathematics and Economics, vol. 9, no. 2-3, pp. 127-130, 1990.

[7] E. W. Frees, "Nonparametric estimation of probability of ruin," Astin Bulletin, vol. 16, no. 3, pp. 81-90, 1986.

[8] R. Mnatsakanov, L. L. Ruymgaart, and F. H. Ruymgaart, "Nonparametric estimation of ruin probabilities given a random sample of claims," Mathematical Methods of Statistics, vol. 17, no. 1, pp. 35-43, 2008.

[9] S. M. Pitts, "Nonparametric estimation of compound distributions with applications in insurance," Annals of the Institute of Statistical Mathematics, vol. 46, no. 3, pp. 537-555, 1994.

[10] K. Politis, "Semiparametric estimation for non-ruin probabilities," Scandinavian Actuarial Journal, vol. 2003, no. 1, pp. 75-96, 2003.

[11] H. You, J. Guo, and J. Jiang, "Interval estimation of the ruin probability in the classical compound Poisson risk model," Computational Statistics and Data Analysis, vol. 144, Article ID 106890, 2020.

[12] Z. Zhang, H. Yang, and H. Yang, "On a nonparametric estimator for ruin probability in the classical risk model," Scandinavian Actuarial Journal, vol. 2014, no. 4, pp. 309-338, 2014.

[13] F. Comte and V. Genon-Catalot, "Nonparametric estimation for pure jump Lévy processes based on high frequency data," Stochastic Processes and Their Applications, vol. 119, no. 12, pp. 4088-4123, 2009.

[14] F. Comte and V. Genon-Catalot, "Estimation for Lévy processes from high frequency data within a long time interval," The Annals of Statistics, vol. 39, no. 2, pp. 803-837, 2011.

[15] Z. Zhang and H. Yang, "Nonparametric estimate of the ruin probability in a pure-jump Lévy risk model," Insurance: Mathematics and Economics, vol. 53, no. 1, pp. 24-35, 2013.

[16] Y. Shimizu and Z. Zhang, "Estimating Gerber-Shiu functions from discretely observed Lévy driven surplus," Insurance: Mathematics and Economics, vol. 74, pp. 84-98, 2017.

[17] H. You and C. Cai, "Nonparametric estimation for a spectrally negative Lévy process based on high frequency data," Journal of Computational and Applied Mathematics, vol. 345, pp. 196-205, 2019.

[18] C. Cai, N. Chen, and H. You, "Nonparametric estimation for a spectrally negative Lévy risk process based on low-frequency observation," Journal of Computational and Applied Mathematics, vol. 328, pp. 432-442, 2018.

[19] C. Mancini, "Estimation of the characteristics of the jumps of a general Poisson-diffusion model," Scandinavian Actuarial Journal, vol. 2004, no. 1, pp. 42-52, 2004.

[20] C. Mancini, "Non-parametric threshold estimation for models with stochastic diffusion coefficient and jumps," Scandinavian Journal of Statistics, vol. 36, no. 2, pp. 270-296, 2009.

[21] Y. Shimizu, "A new aspect of a risk process and its statistical inference," Insurance: Mathematics and Economics, vol. 44, no. 1, pp. 70-77, 2009. 
[22] Y. Shimizu, "Functional estimation for Lévy measures of semimartingales with Poissonian jumps," Journal of Multivariate Analysis, vol. 100, no. 6, pp. 1073-1092, 2009.

[23] M. Huzak, M. Perman, H. Š rma, and Z. Vondraček, "Ruin probabilities for general perturbed risk processes," The Annals of Applied Probability, vol. 14, no. 3, pp. 1378-1397, 2004.

[24] D. E. Chauveau, A. C. M. Vanrooij, and F. H. Ruymgaart, "Regularized inversion of noisy Laplace transforms," $A d$ vances in Applied Mathematics, vol. 15, no. 2, pp. 186-201, 1994.

[25] Z. Zhang and H. Yang, "Nonparametric estimation for the ruin probability in a Lévy risk model under low-frequency observation," Insurance: Mathematics and Economics, vol. 59, pp. 168-177, 2014.

[26] Z. Zhang and W. Su, "Estimating the Gerber-Shiu function in a Lévy risk model by Laguerre series expansion," Journal of Computational and Applied Mathematics, vol. 346, pp. 133149, 2019.

[27] Z. Zhang and W. Su, "A new efficient method for estimating the Gerber-Shiu function in the classical risk model," Scandinavian Actuarial Journal, vol. 2018, no. 5, pp. 426-449, 2018.

[28] W. Yu, Y. Yong, G. Guan, Y. Huang, W. Su, and C. Cui, "Valuing guaranteed minimum death benefits by cosine series expansion," Mathematics, vol. 7, no. 9, p. 835, 2019.

[29] X. Peng, W. Su, W. Su, and Z. Zhang, "On a perturbed compound Poisson risk model under a periodic thresholdtype dividend strategy," Journal of Industrial \& Management Optimization, vol. 16, no. 4, pp. 1967-1986, 2020.

[30] W. Yu, P. Guo, Q. Wang et al., "On a periodic capital injection and barrier dividend strategy in the compound Poisson risk model," Mathematics, vol. 8, no. 4, p. 511, 2020.

[31] I. Vondraček and S. F. Shreve, Brownian Motion and Stochastic Calculus, Springer, New York, NY, USA, 1999. 